\title{
A Method for Simulating Burr Type III and Type XII Distributions through $L$-Moments and $L$-Correlations
}

\author{
Mohan D. Pant ${ }^{1}$ and Todd C. Headrick ${ }^{2}$ \\ ${ }^{1}$ Department of Curriculum and Instruction, 320-B Science Hall, University of Texas at Arlington, Arlington, TX 76019, USA \\ ${ }^{2}$ Section on Statistics and Measurement, Department of EPSE, Southern Illinois University Carbondale, 222-J Wham Bldg, Carbondale, \\ IL 62901-4618, USA
}

Correspondence should be addressed to Mohan D. Pant; mpant@uta.edu

Received 27 January 2013; Accepted 27 March 2013

Academic Editors: F. Ding, E. Skubalska-Rafajlowicz, and F. Zirilli

Copyright (c) 2013 M. D. Pant and T. C. Headrick. This is an open access article distributed under the Creative Commons Attribution License, which permits unrestricted use, distribution, and reproduction in any medium, provided the original work is properly cited.

\begin{abstract}
This paper derives the Burr Type III and Type XII family of distributions in the contexts of univariate $L$-moments and the $L$ correlations. Included is the development of a procedure for specifying nonnormal distributions with controlled degrees of $L$-skew, $L$-kurtosis, and $L$-correlations. The procedure can be applied in a variety of settings such as statistical modeling (e.g., forestry, fracture roughness, life testing, operational risk, etc.) and Monte Carlo or simulation studies. Numerical examples are provided to demonstrate that $L$-moment-based Burr distributions are superior to their conventional moment-based analogs in terms of estimation and distribution fitting. Evaluation of the proposed procedure also demonstrates that the estimates of $L$-skew, $L$-kurtosis, and $L$-correlation are substantially superior to their conventional product moment-based counterparts of skew, kurtosis, and Pearson correlations in terms of relative bias and relative efficiency-most notably when heavy-tailed distributions are of concern.
\end{abstract}

\section{Introduction}

Burr [1] introduced twelve cumulative distribution functions (cdfs) with the primary purpose of fitting distributions to real-world data. Two popular Burr cdfs are referred to as the Burr Type III and Type XII distributions. The specific forms of these two cdfs are given as [1, Equations (11), (20)]

$$
\begin{gathered}
F(x)_{(\text {Type III) }}=\left(1+x^{c}\right)^{-k}, \\
F(x)_{(\text {Type XII) }}=1-\left(1+x^{c}\right)^{-k},
\end{gathered}
$$

where $x \in(0, \infty), c$, and $k$ are real-valued parameters that determine the mean, variance, skew, and kurtosis of a distribution. The parameter $c$ is negative for the Type III and positive for the Type XII distributions, whereas the parameter $k$ is positive for both the distributions [2].

The Burr Type III and Type XII distributions attract special attention because they include several families of nonnormal distributions (e.g., the Gamma distribution) with varying degrees of skew and kurtosis [2-5]. Further, these distributions have been used in a variety of applied mathematics contexts. Some examples include modeling events associated with forestry [6,7], fracture roughness $[8,9]$, life testing [10, 11], operational risk [12], option market price distributions [13], meteorology [14], modeling crop prices [15], software reliability growth [16], reliability analysis [17], and in the context of Monte Carlo or simulation studies (e.g., [2]).

The quantile functions associated with (1) are expressed as [2, Equations (5), (6)]

$$
\begin{gathered}
q(u)_{\text {(Type III) }}=F^{-1}(x)=\left(u^{-1 / k}-1\right)^{1 / c}, \\
q(u)_{\text {(Type XII) }}=F^{-1}(x)=\left((1-u)^{-1 / k}-1\right)^{1 / c},
\end{gathered}
$$

where $u \sim$ iid $U(0,1)$ with pdf and $\operatorname{cdf}$ as 1 and $u$, respectively. The shape of a Burr distribution associated with (2) or (3) is contingent on the values of the shape parameters ( $c$ and $k$ ), which can be determined by simultaneously solving equations (16) and (17) from [2, p. 2211] for given values of skew and kurtosis. 


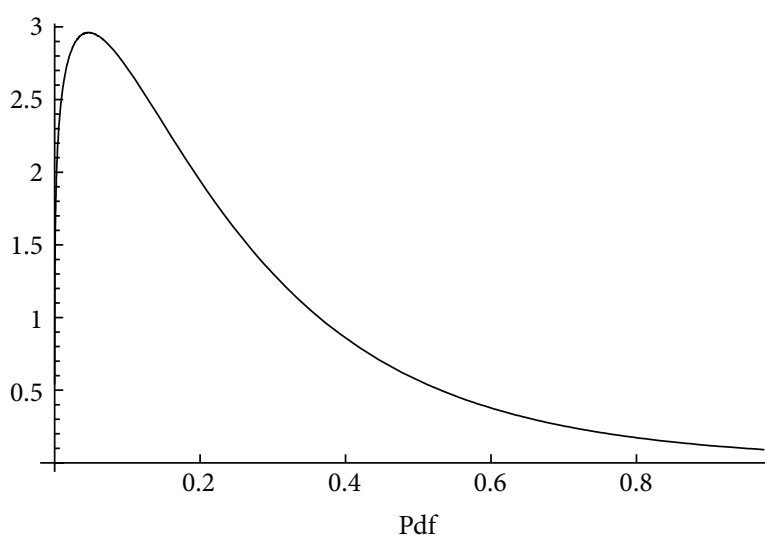

(a)

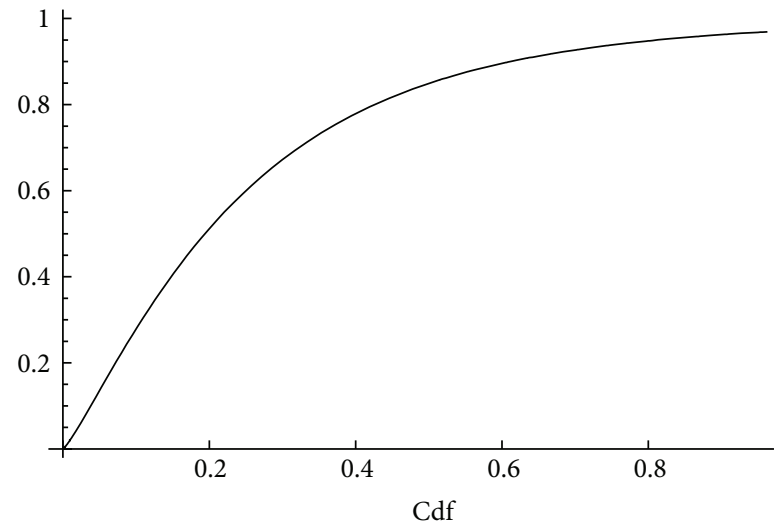

(b)

Figure 1: The pdf and cdf of the Burr Type XII distribution with skew $\left(\gamma_{3}\right)=3$ and kurtosis $\left(\gamma_{4}\right)=21$. The solved values of $c$ and $k$ used in (5) and (1) are $c=1.187185$ and $k=5.199500$ which are also associated with the parameters in Tables 1 and 2.

In order for (2) or (3) to produce a valid Burr pdf, the quantile function $q(u)$ is required to be a strictly increasing monotone function [2]. This requirement implies that an inverse function $\left(q^{-1}\right)$ exists. As such, the cdf associated with (2) or (3) can be expressed as $F(q(u))=F(u)=u$, and subsequently differentiating this cdf with respect to $u$ will yield the parametric form of the pdf for $q(u)$ as $f(q(u))=$ $1 / q^{\prime}(u)$. We would also note that the simple closed form expressions for the pdfs associated with (1) can be given as [1]

$$
\begin{aligned}
& f(x)_{(\text {Type III })}=-c k x^{c-1}\left(1+x^{c}\right)^{-(k+1)}, \\
& f(x)_{(\text {Type XII) }}=c k x^{c-1}\left(1+x^{c}\right)^{-(k+1)} .
\end{aligned}
$$

Some of the problems associated with conventional moment-based estimates are that they can be (a) substantially biased, (b) highly dispersed, or (c) influenced by outliers $[18,19]$ and thus may not be good representatives of the true parameters. To demonstrate, Figure 1 gives the graphs of the pdf and cdf associated with Burr Type XII distribution with skew $\left(\gamma_{3}\right)=3$ and kurtosis $\left(\gamma_{4}\right)=21$. These values of skew and kurtosis have been used in a number of studies [18, 20-22]. Table 1 gives the parameters and sample estimates of skew and kurtosis for the distribution in Figure 1. Inspection of Table 1 indicates that the bootstrap estimates $\left(g_{3}\right.$ and $\left.g_{4}\right)$ of skew and kurtosis $\left(\gamma_{3}\right.$ and $\left.\gamma_{4}\right)$ are substantially attenuated below their corresponding parameter values with greater bias and variance as the order of the estimate increases. Specifically, for sample size of $n=50$, the values of the estimates are only $63.53 \%$, and $22.81 \%$ of their corresponding parameters, respectively. The estimates $\left(g_{3}\right.$ and $\left.g_{4}\right)$ of skew and kurtosis $\left(\gamma_{3}\right.$ and $\left.\gamma_{4}\right)$ in Table 1 were calculated based on Fisher's $k$ statistics formulae (see, e.g., [23, pages 299-300]), currently used by most commercial software packages such as SAS, SPSS, and Minitab, and so forth, for computing the values of skew and kurtosis (where $\gamma_{3,4}=0$ for the standard normal distribution).
TABLE 1: Conventional moment-based parameters $\left(\gamma_{3}, \gamma_{4}\right)$ of skew and kurtosis and their estimates $\left(g_{3}, g_{4}\right)$ for the pdf in Figure 1 . Each bootstrapped estimate (Estimate), associated 95\% bootstrap confidence interval (95\% Bootstrap C.I.), and the standard error (St. error) were based on resampling 25,000 statistics. Each statistic was based on a sample size of $n=50$.

\begin{tabular}{lccc}
\hline Parameter & Estimate & 95\% Bootstrap C.I. & St. error \\
\hline$\gamma_{3}=3.00$ & $g_{3}=1.9060$ & $(1.8955,1.9163)$ & 0.0053 \\
$\gamma_{4}=21.00$ & $g_{4}=4.7900$ & $(4.7199,4.8525)$ & 0.0338 \\
\hline
\end{tabular}

Another unfavorable quality of conventional momentbased estimators of skew and kurtosis is that their values are algebraically bounded by the sample size $(n)$ such that $\left|g_{3}\right| \leq \sqrt{n}$ and $g_{4} \leq n$ [18]. This constraint implies that if a researcher wants to simulate non-normal data with kurtosis $\gamma_{4}=21$ as in Table 1 , and drawing a sample of size $n=15$ from this population, the largest possible value of the computed estimate $\left(g_{4}\right)$ of kurtosis $\left(\gamma_{4}\right)$ is only 15 , which is only $71.43 \%$ of the parameter.

The method of $L$-moments introduced by Hosking [19] is an attractive alternative to conventional moments and can be used for describing theoretical probability distributions, fitting distributions to real-world data, estimating parameters, and testing of hypotheses $[18,19,24,25]$. In these contexts, we note that the $L$-moment based estimators of $L$-skew, $L$ kurtosis, and $L$-correlation have been introduced to address the limitations associated with conventional moment-based estimators [18, 19, 24-31]. Some qualities of $L$-moments that make them superior to conventional moments are that they (a) exist for any distribution with finite mean, and their estimates are (b) nearly unbiased for any sample size and less affected from sampling variability, (c) more robust in the presence of outliers in the sample data, and (d) are not algebraically bounded by the sample size $[18,19,24,25]$. For example, the estimates $\left(t_{3}\right.$ and $\left.t_{4}\right)$ of $L$-skew and $L$ kurtosis $\left(\tau_{3}\right.$ and $\left.\tau_{4}\right)$ in Table 2 are relatively closer to their respective parameter values with much smaller variance 
TABLE 2: $L$-moment-based parameters $\left(\tau_{3}, \tau_{4}\right)$ of $L$-skew and $L$ kurtosis and their estimates $\left(t_{3}, t_{4}\right)$ for the pdf in Figure 1. Each bootstrapped estimate (Estimate), associated 95\% bootstrap confidence interval (95\% Bootstrap C.I.), and the standard error (St. error) were based on resampling 25,000 statistics. Each statistic was based on a sample size of $n=50$.

\begin{tabular}{lccc}
\hline Parameter & Estimate & 95\% Bootstrap C.I. & St. error \\
\hline$\tau_{3}=0.3536$ & $t_{3}=0.3455$ & $(0.3445,0.3465)$ & 0.0005 \\
$\tau_{4}=0.2020$ & $t_{4}=0.1965$ & $(0.1955,0.1975)$ & 0.0005
\end{tabular}

compared to their conventional moment-based counterparts as in Table 1. Inspection of Table 2 shows that for the sample size of $n=50$, the values of the estimates are on average $97.71 \%$ and $97.28 \%$ of their corresponding parameters.

In view of the above, the main purpose of this study is to characterize the Burr Type III and Type XII distributions through the method of $L$-moments in order to obviate the problems associated with conventional moment-based estimators. Another purpose of this study is to develop an $L$ correlation-based methodology to simulate correlated Burr Type III and Type XII distributions. Specifically, in Section 2, a brief introduction to univariate $L$-moments is provided. The systems of equations associated with the Type III and Type XII distributions are subsequently derived for determining the shape parameters ( $c$ and $k$ ) for user specified values of $L$-skew $\left(\tau_{3}\right)$ and $L$-kurtosis $\left(\tau_{4}\right)$. In Section 3, a comparison between conventional and $L$-moment-based Burr Type III and Type XII distributions is presented in the contexts of estimation and distribution fitting. Numerical examples based on Monte Carlo simulation are also provided to confirm the methodology and demonstrate the advantages that $L$ moments have over conventional moments. In Section 4, an introduction to the coefficient of $L$-correlation is provided, and the methodology for solving for intermediate correlations for specified $L$-correlation structure is subsequently presented. In Section 5, the steps for implementing the proposed $L$-moment procedure are described for simulating non-normal Burr Type III and Type XII distributions with controlled skew ( $L$-skew), kurtosis ( $L$-kurtosis), and Pearson correlations ( $L$-correlations). Numerical examples and the results of simulation are also provided to confirm the derivations and compare the new procedure with the conventional moment-based procedure. In Section 6, the results of the simulation are discussed.

\section{Methodology}

2.1. Theoretical and Empirical Definitions of L-Moments. $L$-moments can be expressed as certain linear combinations of probability weighted moments (PWMs). Let $X_{1}, \ldots, X_{i}, \ldots, X_{n}$ be identically and independently distributed random variables each with pdf $f(x), \operatorname{cdf} F(x)$, and the quantile function $F^{-1}(x)$, then the PWMs are defined as [18, Equation (6)]

$$
\beta_{r}=\int F^{-1}(x)\{F(x)\}^{r} f(x) d x,
$$

where $r=0,1,2,3$. The first four $L$-moments $\left(\lambda_{i=1, \ldots, 4}\right)$ associated with $X$ can be expressed in simplified forms as [25, pages 20-22]

$$
\begin{gathered}
\lambda_{1}=\beta_{0}, \\
\lambda_{2}=2 \beta_{1}-\beta_{0}, \\
\lambda_{3}=6 \beta_{2}-6 \beta_{1}+\beta_{0}, \\
\lambda_{4}=20 \beta_{3}-30 \beta_{2}+12 \beta_{1}-\beta_{0},
\end{gathered}
$$

where the coefficients associated with $\beta_{r=0, \ldots, 3}$ in (7) are obtained from shifted orthogonal Legendre polynomials and are computed as in [25, pages 20-22] or in [18, pages 4-5].

The notations $\lambda_{1}$ and $\lambda_{2}$ denote the location and scale parameters. Specifically, in the literature of $L$-moments, $\lambda_{1}$ is referred to as the $L$-location parameter which is equal to the arithmetic mean, and $\lambda_{2}(>0)$ is referred to as the $L$ scale parameter and is one-half of Gini's coefficient of mean difference [23, pages 47-48]. Dimensionless $L$-moment ratios are defined as the ratios of higher-order $L$-moments (i.e., $\lambda_{3}$ and $\lambda_{4}$ ) to $\lambda_{2}$. Thus, $\tau_{3}=\lambda_{3} / \lambda_{2}$ and $\tau_{4}=\lambda_{4} / \lambda_{2}$ are, respectively, the indices of $L$-skew and $L$-kurtosis. In general, the indices of $L$-skew and $L$-kurtosis are bounded in the interval $-1<\tau_{3,4}<1$, and as in conventional moment theory, a symmetric distribution has $L$-skew equal to zero [18]. The boundary region for $L$-skew $\left(\tau_{3}\right)$ and $L$-kurtosis $\left(\tau_{4}\right)$ for a continuous distribution is given by the inequality (see, [32])

$$
\frac{5 \tau_{3}^{2}-1}{4}<\tau_{4}<1 .
$$

Empirical $L$-moments for a sample (of size $n$ ) of realworld data are expressed as linear combinations of the unbiased estimators of the PWMs based on sample order statistics $X_{1: n} \leq X_{2: n} \leq \cdots \leq X_{n: n}$. Specifically, the unbiased estimators of the PWMs are given as [19, pages 113-114]

$$
b_{r}=\frac{1}{n} \sum_{i=r+1}^{n} \frac{(i-1)(i-2) \cdots(i-r)}{(n-1)(n-2) \cdots(n-r)} X_{i: n}
$$

where $r=0,1,2,3$ and $b_{0}$ is the sample mean. The first four sample $L$-moments $\left(\ell_{1}, \ell_{2}, \ell_{3}, \ell_{4}\right)$ are obtained by substituting $b_{r}$ from (9) instead of $\beta_{r}$ in (7). The sample $L$-moment ratios (i.e., $L$-skew and $L$-kurtosis) are denoted by $t_{3}$ and $t_{4}$, where $t_{3}=\ell_{3} / \ell_{2}$ and $t_{4}=\ell_{4} / \ell_{2}$.

2.2. L-Moments for Burr Type III Distributions. Substituting $F^{-1}(x)=\left(u^{-1 / k}-1\right)^{1 / c}$ from $(2), F(x)=u$, and $f(x)=1$ in (6), the $r$ th PWM for the Burr Type III distributions is given by

$$
\beta_{r}=\int_{0}^{1}\left(u^{-1 / k}-1\right)^{1 / c} u^{r} d u .
$$

Equation (10) can also be expressed as

$$
\beta_{r}=\int_{0}^{1} k\left(u^{1 / k}\right)^{(k+k r-1 / c)-1}\left(1-u^{1 / k}\right)^{1 / c}\left(k^{-1} u^{(1 / k)-1} d u\right) .
$$


Let $u^{1 / k}=x$. Then, $k^{-1} u^{(1 / k)-1} d u=d x$. Substituting in (11), the $r$ th PWM can be expressed as

$$
\beta_{r}=k \int_{0}^{1} x^{(k+k r-1 / c)-1}(1-x)^{(1+1 / c)-1} d x .
$$

The integral in (12) is a beta function, $\operatorname{Beta}(\alpha, \beta)$, where $\alpha=$ $k+k r-1 / c$ and $\beta=1+1 / c$ such that $\operatorname{Real}(\alpha), \operatorname{Real}(\beta)>0$.

Integrating (12) for $\beta_{r=0,1,2,3}$ and substituting these PWMs into (7) and simplifying gives the following system of equations for the Type III distributions:

$$
\begin{aligned}
& \lambda_{1}=\frac{k \Gamma[k-1 / c] \Gamma[1+1 / c]}{\Gamma[1+k]}, \\
& \lambda_{2}=\Gamma\left[1+\frac{1}{c}\right]\left(\frac{\Gamma[2 k-1 / c]}{\Gamma[2 k]}-\frac{\Gamma[k-1 / c]}{\Gamma[k]}\right), \\
& \tau_{3}=\left\{\Gamma [ 1 + 3 k ] \left(6 \Gamma[1+k] \Gamma\left[2 k-\frac{1}{c}\right]\right.\right. \\
& \left.-\Gamma[1+2 k] \Gamma\left[k-\frac{1}{c}\right]\right) \\
& \left.-6 \Gamma[1+k] \Gamma[1+2 k] \Gamma\left[3 k-\frac{1}{c}\right]\right\} \\
& \times\left\{\Gamma[1+3 k] \times\left(\Gamma[1+2 k] \Gamma\left[k-\frac{1}{c}\right]\right.\right. \\
& \left.\left.-2 \Gamma[1+k] \Gamma\left[2 k-\frac{1}{c}\right]\right)\right\}^{-1}, \\
& \tau_{4}=\left\{\Gamma[1+4 k] \times\left\{\Gamma[1+3 k] \times\left(\Gamma[1+2 k] \Gamma\left[k-\frac{1}{c}\right]\right.\right.\right. \\
& \left.-12 \Gamma[1+k] \Gamma\left[2 k-\frac{1}{c}\right]\right) \\
& \left.+30 \Gamma[1+k] \Gamma[1+2 k] \Gamma\left[3 k-\frac{1}{c}\right]\right\} \\
& \left.-20 \Gamma[1+k] \Gamma[1+2 k] \Gamma[1+3 k] \Gamma\left[4 k-\frac{1}{c}\right]\right\} \\
& \times\left\{\Gamma[1+3 k] \times \Gamma[1+4 k] \times\left(\Gamma[1+2 k] \Gamma\left[k-\frac{1}{c}\right]\right.\right. \\
& \left.\left.-2 \Gamma[1+k] \Gamma\left[2 k-\frac{1}{c}\right]\right)\right\}^{-1} \text {. }
\end{aligned}
$$

2.3. L-Moments for the Burr Type XII Distributions. Substituting $F^{-1}(x)=\left((1-u)^{-1 / k}-1\right)^{1 / c}$ from (3), $F(x)=u$, and $f(x)=1$ in (6), the $r$ th PWM for the Burr Type XII distributions is given by

$$
\beta_{r}=\int_{0}^{1}\left((1-u)^{-1 / k}-1\right)^{1 / c} u^{r} d u
$$

After some manipulations, (16) can be expressed as

$$
\begin{aligned}
\beta_{r}=\int_{0}^{1}-k u^{r}\left((1-u)^{1 / k}\right)^{(k-1 / c)-1}\left(1-(1-u)^{1 / k}\right)^{1 / c} \\
\times\left(-k^{-1}(1-u)^{(1 / k)-1} d u\right) .
\end{aligned}
$$

Let $(1-u)^{1 / k}=x$. Then, $-k^{-1}(1-u)^{(1 / k)-1} d u=d x$. Substituting in (17), the $r$ th PWM can be rewritten as

$$
\beta_{r}=k \int_{0}^{1}\left(1-x^{k}\right)^{r} x^{(k-1 / c)-1}(1-x)^{(1+1 / c)-1} d x .
$$

Integrating (18) for $\beta_{r=0,1,2,3}$ and substituting these PWMs into (7) and simplifying gives the following system of equations for the Type XII distributions:

$$
\begin{aligned}
& \lambda_{1}=\frac{k \Gamma[k-1 / c] \Gamma[1+1 / c]}{\Gamma[1+k]}, \\
& \lambda_{2}=-\Gamma\left[1+\frac{1}{c}\right]\left(\frac{\Gamma[2 k-1 / c]}{\Gamma[2 k]}-\frac{\Gamma[k-1 / c]}{\Gamma[k]}\right) \\
& \tau_{3}=\left\{\Gamma [ 1 + 3 k ] \left(-6 \Gamma[1+k] \Gamma\left[2 k-\frac{1}{c}\right]\right.\right. \\
& \left.+\Gamma[1+2 k] \Gamma\left[k-\frac{1}{c}\right]\right) \\
& \left.+6 \Gamma[1+k] \Gamma[1+2 k] \Gamma\left[3 k-\frac{1}{c}\right]\right\} \\
& \times\left\{\Gamma[1+3 k] \times\left(\Gamma[1+2 k] \Gamma\left[k-\frac{1}{c}\right]\right.\right. \\
& \left.\left.-2 \Gamma[1+k] \Gamma\left[2 k-\frac{1}{c}\right]\right)\right\}^{-1}, \\
& \tau_{4}=\{\Gamma[k] \Gamma[1+4 k] \\
& \times\left\{\Gamma[1+3 k] \times\left\{\Gamma[1+2 k] \Gamma\left[k-\frac{1}{c}\right]\right.\right. \\
& \left.-12 \Gamma[1+k] \Gamma\left[2 k-\frac{1}{c}\right]\right\} \\
& \left.+30 \Gamma[1+k] \Gamma[1+2 k] \Gamma\left[3 k-\frac{1}{c}\right]\right\} \\
& \left.-40 \Gamma[2 k] \Gamma[1+k]^{2} \Gamma[1+3 k] \Gamma\left[4 k-\frac{1}{c}\right]\right\} \\
& \times\{\Gamma[k] \Gamma[1+3 k] \times \Gamma[1+4 k] \\
& \left.\times\left\{\Gamma[1+2 k] \Gamma\left[k-\frac{1}{c}\right]-2 \Gamma[1+k] \Gamma\left[2 k-\frac{1}{c}\right]\right\}\right\}^{-1} .
\end{aligned}
$$

Given specified values of $L$-skew $\left(\tau_{3}\right)$ and $L$-kurtosis $\left(\tau_{4}\right)$ the systems of equations (15) and (21) can be simultaneously solved for real values of $c$ and $k$. The solved values of $c$ and $k$ can be substituted in (2) and (3), respectively, for generating the Burr Type III and Type XII distributions. Further, the solved values of $c$ and $k$ can be substituted in (13)-(14) and (19)-(20), respectively, for computing the values of $L$-mean $\left(\lambda_{1}\right)$ and $L$-scale $\left(\lambda_{2}\right)$ associated with the Type III and Type XII distributions. In the next section, two examples are provided to demonstrate the aforementioned methodology and the advantages that $L$-moments have over 
TABLE 3: Chi-square goodness of fit statistics for the conventional $(C)$ moment- and the $L$-moment- $(L)$ based Burr Type XII approximations for the ankle circumference $(n=252)$ data in Figure 3.

\begin{tabular}{lccccc}
\hline Percent & Expected & Obs. $(C)$ & Obs. $(L)$ & Ankle circumference $(C)$ & Ankle circumference $(L)$ \\
\hline 10 & 25.2 & 22 & 18 & $<21.4866$ & $<21.3760$ \\
20 & 25.2 & 15 & 29 & $21.4866-21.7890$ & $21.3760-21.8701$ \\
30 & 25.2 & 29 & 30 & $21.7890-22.0747$ & $21.8701-22.2360$ \\
40 & 25.2 & 18 & 26 & $22.0747-22.3687$ & $22.2360-22.5606$ \\
50 & 25.2 & 30 & 25 & $22.3687-22.6888$ & $22.5606-22.8795$ \\
60 & 25.2 & 27 & 27 & $22.6888-23.0571$ & $22.8795-23.2201$ \\
70 & 25.2 & 30 & 20 & $23.0571-23.5107$ & $23.2201-23.6175$ \\
80 & 25.2 & 33 & 32 & $23.5107-24.1310$ & $23.6175-24.1426$ \\
90 & 25.2 & 19 & 20 & $24.1310-25.1858$ & $24.1426-25.0244$ \\
100 & 25.2 & & & 25.1858 or more & 25.0244 or more \\
\hline & & & $\chi^{2}=13.6349$ & $\chi^{2}=7.6825$ \\
& & & & $p=0.0181$ & $p .1746$ \\
\hline
\end{tabular}

TABLE 4: Conventional moment based parameters of the mean $(\mu)$, standard deviation $(\sigma)$, skew $\left(\gamma_{3}\right)$, and kurtosis $\left(\gamma_{4}\right)$ along with their corresponding values of shape parameters $(c$ and $k$ ) for the four distributions (dashed curves) in Figure 2 (Panel a).

\begin{tabular}{lcccccc}
\hline Distribution & $\mu$ & $\sigma$ & $\gamma_{3}$ & $\gamma_{4}$ & $c$ \\
\hline 1 & 0.306182 & 0.362801 & 4.221159 & 59.454545 & 1.097740 & 4.481719 \\
2 & 0.139427 & 0.263634 & 2.828427 & 12.0 & -5.542062 & 0.027237 \\
3 & 0.415523 & 0.242125 & 1.139547 & 2.4 & 2.025127 \\
4 & 0.937510 & 0.139248 & 0.0 & 1.2 & -15.776368 & 0.540152 \\
\hline
\end{tabular}

conventional moments in the contexts of estimation and distribution fitting.

\section{Comparison of $L$-Moments with Conventional Moments}

3.1. Estimation. An example to demonstrate the advantages of $L$-moment-based estimation over conventional momentbased estimation is provided in Figure 2 and Tables 4-7. Given in Figure 2 are the pdfs of the $F$ distribution $(3,10)$, Chi-square $(d f=1)$, extreme value $(0,1)$, and logistic $(0,1)$ distributions superimposed, respectively, by the Burr Type XII, Type III, Type XII, and Type III pdfs (dashed curves) in both (a) conventional moment- and (b) L-momentbased systems. The conventional moment-based parameters of skew $\left(\gamma_{3}\right)$ and kurtosis $\left(\gamma_{4}\right)$ associated with these four distributions, given in Table 4 , were computed by using equations (11)-(13) from [2, page 2211]. The values of shape parameters ( $c$ and $k$ ) given in Table 4 were determined by solving equations (16) and (17) from [2, page 2211]. The values of $c$ and $k$ were used in (4) and (5) to superimpose the conventional moment-based Burr Type XII, Type III, Type XII, and Type III distributions as shown in Figure 2(a).

The $L$-moment-based parameters of $L$-skew $\left(\tau_{3}\right)$ and $L$-kurtosis $\left(\tau_{4}\right)$ associated with the four distributions in Figure 2, given in Table 5, were obtained in three steps as follows: (a) compute the values of PWMs $\left(\beta_{r=0,1,2,3}\right)$ using (6), (b) substitute these PWMs into (7) to obtain the values of the first four $L$-moments, and (c) compute the values of $\tau_{3}$ and $\tau_{4}$ using $\tau_{3}=\lambda_{3} / \lambda_{2}$ and $\tau_{4}=\lambda_{4} / \lambda_{2}$. The values of shape parameters ( $c$ and $k$ ) given in Table 5 were determined by solving the systems of equations (15) and (21), respectively. These values of $c$ and $k$ were used in (4) and (5) to superimpose the $L$-moment-based Burr Type XII, Type III, Type XII, and Type III distributions as shown in Figure 2(b).

To superimpose the Type III or Type XII distribution, the quantile function $q(u)$ in (2) or (3) was transformed into (a) $(\bar{X} \sigma-\mu S+S q(u)) / \sigma$, and (b) $\left(\ell_{1} \lambda_{2}-\lambda_{1} \ell_{2}+\ell_{2} q(u)\right) /$ $\lambda_{2}$, respectively, where $(\bar{X}, S)$ and $(\mu, \sigma)$ are the values of mean, standard deviation, whereas $\left(\ell_{1}, \ell_{2}\right)$ and $\left(\lambda_{1}, \lambda_{2}\right)$ are the values of $L$-mean, $L$-scale obtained from the original distribution and the respective Burr Type III or Type XII approximation, respectively.

The advantages of $L$-moment-based estimators over those based on conventional moments can also be demonstrated in the context of Burr Type III and Type XII distributions by considering the Monte Carlo simulation results associated with the indices for the percentage of relative bias (RB\%) and standard error (St. error) reported in Tables 6 and 7.

Specifically, a Fortran [33] algorithm was written to simulate 25,000 independent samples of sizes $n=25$ and $n=$ 1000 , and the conventional moment-based estimates $\left(g_{3}\right.$ and $\left.g_{4}\right)$ of skew and kurtosis $\left(\gamma_{3}\right.$ and $\left.\gamma_{4}\right)$ and the $L$-moment based estimates $\left(t_{3}\right.$ and $\left.t_{4}\right)$ of $L$-skew and $L$-kurtosis $\left(\tau_{3}\right.$ and $\tau_{4}$ ) were computed for each of the $(2 \times 25,000)$ samples based on the parameters and the values of $c$ and $k$ listed in Tables 4 and 5. The estimates $\left(g_{3}\right.$ and $\left.g_{4}\right)$ of $\gamma_{3}$ and $\gamma_{4}$ were computed based on Fisher's $k$-statistics formulae [23, pages 47-48], whereas the estimates $\left(t_{3}\right.$ and $\left.t_{4}\right)$ of $\tau_{3}$ and $\tau_{4}$ were computed using (7) and (9). Bias-corrected accelerated bootstrapped average 
TABLE 5: $L$-moment-based parameters of $L$-mean $\left(\lambda_{1}\right), L$-scale $\left(\lambda_{2}\right), L$-skew $\left(\tau_{3}\right)$, and $L$-kurtosis $\left(\tau_{4}\right)$ along with their corresponding values of shape parameters ( $c$ and $k$ ) for the four distributions (dashed curves) in Figure 2 (Panel $b$ ).

\begin{tabular}{lcccccc}
\hline Distribution & $\lambda_{1}$ & $\lambda_{2}$ & $\tau_{3}$ & $\tau_{4}$ & $c$ \\
\hline 1 & 0.441134 & 0.221816 & 0.387355 & 0.233548 & 1.227122 & 3.417227 \\
2 & 0.349660 & 0.226233 & 0.464102 & 0.226497 & -2.944030 & 0.126960 \\
3 & 0.643787 & 0.182777 & 0.169925 & 0.150375 & 2.498521 & 2.913452 \\
4 & 0.936479 & 0.085223 & 0.0 & 0.166667 & -13.875566 & 0.562179 \\
\hline
\end{tabular}

TABLE 6: Skew $\left(\gamma_{3}\right)$ and kurtosis $\left(\gamma_{4}\right)$ results for the conventional moment procedure.

\begin{tabular}{|c|c|c|c|c|c|}
\hline Distribution & Parameter & Estimate & 95\% Bootstrap C.I. & St. error & $\mathrm{RB} \%$ \\
\hline \multicolumn{6}{|c|}{$n=25$} \\
\hline \multirow{2}{*}{1} & $\gamma_{3}=4.2212$ & $g_{3}=1.75$ & $1.7397,1.7597$ & 0.00510 & -58.54 \\
\hline & $\gamma_{4}=59.4545$ & $g_{4}=3.34$ & $3.2911,3.3871$ & 0.02430 & -94.38 \\
\hline \multirow{2}{*}{2} & $\gamma_{3}=2.8284$ & $g_{3}=2.151$ & $2.1428,2.1596$ & 0.00429 & -23.95 \\
\hline & $\gamma_{4}=12.0$ & $g_{4}=4.215$ & $4.1715,4.2614$ & 0.02306 & -64.88 \\
\hline \multirow{2}{*}{3} & $\gamma_{3}=1.1395$ & $g_{3}=0.7929$ & $0.7859,0.7994$ & 0.00346 & -30.42 \\
\hline & $\gamma_{4}=2.4$ & $g_{4}=0.5014$ & $0.4786,0.5220$ & 0.01111 & -79.11 \\
\hline \multirow{2}{*}{4} & $\gamma_{3}=0.0$ & $g_{3}=-0.051$ & $-0.0585,-0.0430$ & 0.00391 & - \\
\hline & $\gamma_{4}=1.2$ & $g_{4}=0.3396$ & $0.3243,0.3552$ & 0.00791 & -71.70 \\
\hline \multicolumn{6}{|c|}{$n=1000$} \\
\hline \multirow{2}{*}{1} & $\gamma_{3}=4.2212$ & $g_{3}=3.606$ & $3.5888,3.6275$ & 0.00972 & -14.57 \\
\hline & $\gamma_{4}=59.4545$ & $g_{4}=26.29$ & $25.8657,26.7366$ & 0.22370 & -55.78 \\
\hline \multirow{2}{*}{2} & $\gamma_{3}=2.8284$ & $g_{3}=2.761$ & $2.7548,2.7681$ & 0.00335 & -2.38 \\
\hline & $\gamma_{4}=12.0$ & $g_{4}=10.17$ & $10.0615,10.3107$ & 0.06265 & -15.25 \\
\hline \multirow{2}{*}{3} & $\gamma_{3}=1.1395$ & $g_{3}=1.124$ & $1.1219,1.1266$ & 0.00122 & -1.36 \\
\hline & $\gamma_{4}=2.4$ & $g_{4}=2.266$ & $2.2451,2.2861$ & 0.01048 & -5.58 \\
\hline \multirow{2}{*}{4} & $\gamma_{3}=0.0$ & $g_{3}=-0.0045$ & $-0.0064,-0.0025$ & 0.00101 & - \\
\hline & $\gamma_{4}=1.2$ & $g_{4}=1.158$ & $1.1487,1.1683$ & 0.00498 & -3.5 \\
\hline
\end{tabular}

TABLE 7: $L$-skew $\left(\tau_{3}\right)$ and $L$-kurtosis $\left(\tau_{4}\right)$ results for the $L$-moment procedure.

\begin{tabular}{|c|c|c|c|c|c|}
\hline Distribution & Parameter & Estimate & 95\% Bootstrap C.I. & St. error & $\mathrm{RB} \%$ \\
\hline \multicolumn{6}{|c|}{$n=25$} \\
\hline \multirow{2}{*}{1} & $\tau_{3}=0.3874$ & $t_{3}=0.3637$ & $0.3621,0.3652$ & 0.00078 & -6.12 \\
\hline & $\tau_{4}=0.2335$ & $t_{4}=0.2149$ & $0.2134,0.2164$ & 0.00078 & -7.97 \\
\hline \multirow{2}{*}{2} & $\tau_{3}=0.4641$ & $t_{3}=0.4446$ & $0.4431,0.4461$ & 0.00076 & -4.20 \\
\hline & $\tau_{4}=0.2265$ & $t_{4}=0.2024$ & $0.2007,0.2042$ & 0.00087 & -10.64 \\
\hline \multirow{2}{*}{3} & $\tau_{3}=0.1699$ & $t_{3}=0.1614$ & $0.1601,0.1627$ & 0.00066 & -5.00 \\
\hline & $\tau_{4}=0.1504$ & $t_{4}=0.1472$ & $0.1462,0.1483$ & 0.00054 & -2.13 \\
\hline \multirow{2}{*}{4} & $\tau_{3}=0.0$ & $t_{3}=-0.0019$ & $-0.0033,-0.0005$ & 0.00071 & - \\
\hline & $\tau_{4}=0.1667$ & $t_{4}=0.1653$ & $0.1643,0.1663$ & 0.00051 & -0.84 \\
\hline \multicolumn{6}{|c|}{$n=1000$} \\
\hline \multirow{2}{*}{1} & $\tau_{3}=0.3874$ & $t_{3}=0.3865$ & $0.3863,0.3868$ & 0.00014 & -0.23 \\
\hline & $\tau_{4}=0.2335$ & $t_{4}=0.2328$ & $0.2325,0.2331$ & 0.00015 & -0.30 \\
\hline \multirow{2}{*}{2} & $\tau_{3}=0.4641$ & $t_{3}=0.4633$ & $0.4630,0.4636$ & 0.00015 & -0.17 \\
\hline & $\tau_{4}=0.2265$ & $t_{4}=0.2255$ & $0.2251,0.2258$ & 0.00019 & -0.44 \\
\hline \multirow{2}{*}{3} & $\tau_{3}=0.1699$ & $t_{3}=0.1696$ & $0.1694,0.1698$ & 0.00010 & -0.18 \\
\hline & $\tau_{4}=0.1504$ & $t_{4}=0.1502$ & $0.1501,0.1504$ & 0.00008 & - \\
\hline \multirow{2}{*}{4} & $\tau_{3}=0.0$ & $t_{3}=-0.0002$ & $-0.0004,0.00002$ & 0.00011 & - \\
\hline & $\tau_{4}=0.1667$ & $t_{4}=0.1667$ & $0.1665,0.1668$ & 0.00007 & - \\
\hline
\end{tabular}



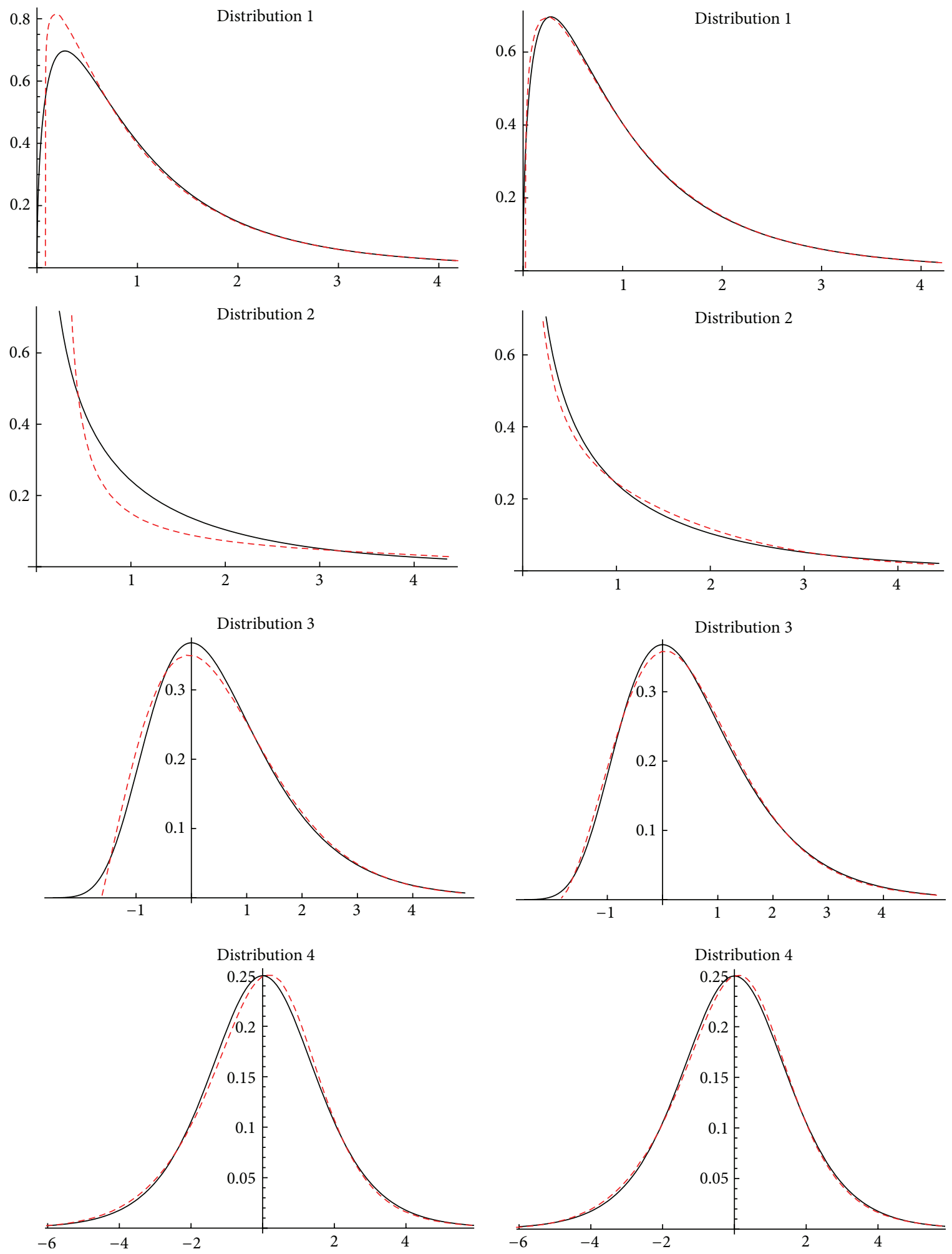

(a)

(b)

FIGURE 2: The pdfs (dashed curves) of the four distributions: Distribution $1:=$ Burr Type XII $\approx F$ distribution $(3,10)$, Distribution $2:=$ Burr Type III $\approx$ Chi square $(d f=1)$, Distribution $3:=$ Burr Type XII $\approx$ Extreme Value $(0,1)$, and Distribution $4:=$ Burr Type III $\approx$ Logistic $(0,1)$ superimposed by the (a) conventional moment- and (b) $L$-moment-based Burr distributions. 


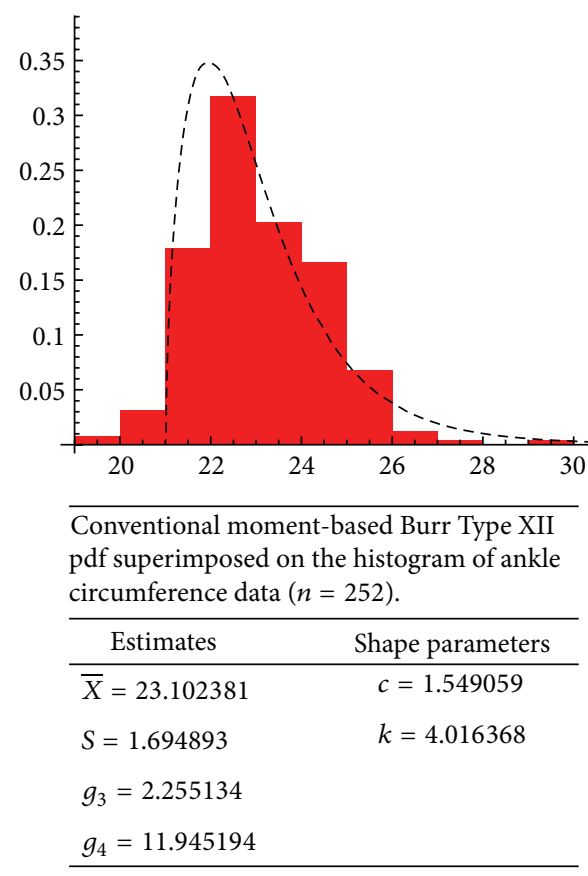

(a)

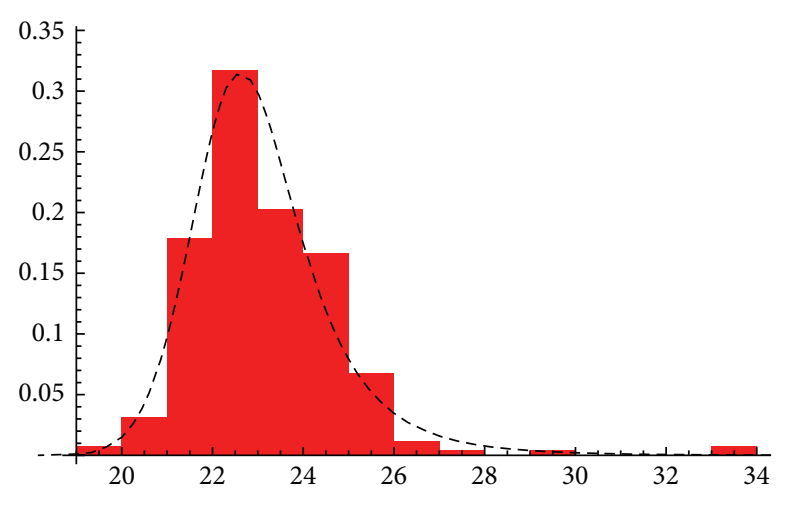

L-moment-based Burr Type XII pdf superimposed on the histogram of ankle circumference data $(n=252)$.

\begin{tabular}{lc}
\hline Estimates & Shape parameters \\
\hline$\ell_{1}=23.102381$ & $c=6.250818$ \\
$\ell_{2}=0.849172$ & $k=0.992608$ \\
$t_{3}=0.161612$ & \\
$t_{4}=0.188653$ & \\
\hline
\end{tabular}

(b)

Figure 3: Histograms of the ankle circumference data superimposed by (a) conventional moment- and (b) L-moment-based Burr Type XII distributions. To superimpose the Burr Type XII distribution (dashed curves), the quantile function $q(u)$ from (3) was transformed as (a) $(\bar{X} \sigma-\mu S+S q(u)) / \sigma$, and (b) $\left(\ell_{1} \lambda_{2}-\lambda_{1} \ell_{2}+\ell_{2} q(u)\right) / \lambda_{2}$, respectively, where $(\bar{X}, S)$ and $(\mu, \sigma)$ are the values of mean and standard deviation, whereas $\left(\ell_{1}, \ell_{2}\right)$ and $\left(\lambda_{1}, \lambda_{2}\right)$ are the values of $L$-mean and $L$-scale obtained from the actual data and the Burr Type XII distribution, respectively.

estimates (Estimate), associated 95\% confidence intervals (95\% Bootstrap C.I.), and standard errors (St. error) were obtained for each type of estimates using 10,000 resamples via the commercial software package Spotfire S+ [34]. Further, if a parameter was outside its associated 95\% bootstrap C.I., then the percentage of relative bias (RB\%) was computed for the estimate as

$$
\mathrm{RB} \%=100 \times \frac{(\text { Estimate }- \text { Parameter })}{\text { Parameter }} .
$$

The results in Tables 6 and 7 illustrate that the $L$-momentbased estimators are superior to their conventional momentbased counterparts in terms of both smaller relative bias and error. These advantages are most pronounced in the context of smaller sample sizes and higher order moments. For example for the Distribution 1, given a sample of size $n=25$, the conventional moment-based estimates $\left(g_{3}\right.$ and $g_{4}$ ) generated in the simulation were, on average, $41.46 \%$ and $5.62 \%$ of their corresponding parameters $\left(\gamma_{3}\right.$ and $\left.\gamma_{4}\right)$. On the other hand, for the same Distribution 1, the $L$-momentbased estimates $\left(t_{3}\right.$ and $\left.t_{4}\right)$ generated in the simulation study were, on average, $93.88 \%$ and $92.03 \%$ of their corresponding parameters $\left(\tau_{3}\right.$ and $\left.\tau_{4}\right)$. Thus, the relative biases of estimators based on $L$-moments are essentially negligible compared to those associated with the estimators based on conventional moments. Also, it can be verified that the standard errors associated with the estimates $t_{3}$ and $t_{4}$ are relatively much smaller and more stable than the standard errors associated with the estimates $g_{3}$ and $g_{4}$.

Inspection of the graphs in Figures 2(a) and 2(b) and the Monte Carlo simulation results in Tables 6 and 7 indicate that the $L$-moment-based Type III and Type XII pdfs provide a more accurate approximation of the four distributions than those based on conventional moment theory.

3.2. Distribution Fitting. Figure 3 shows the conventional moment- and the L-moment-based Burr Type XII pdfs superimposed on the histogram of ankle circumference data obtained from 252 adult males (http://lib.stat.cmu.edu/datasets/bodyfat) as cited in Headrick [35, page 48].

The conventional moment-based estimates $\left(g_{3}\right.$ and $\left.g_{4}\right)$ of skew and kurtosis $\left(\gamma_{3}\right.$ and $\left.\gamma_{4}\right)$ and the $L$-moment-based estimates $\left(t_{3}\right.$ and $\left.t_{4}\right)$ of $L$-skew and $L$-kurtosis $\left(\tau_{3}\right.$ and $\tau_{4}$ ) were computed for the sample of size $n=252$ participants. The estimates of $\gamma_{3}$ and $\gamma_{4}$ were computed based on Fisher's $k$-statistics formulae [23, pages 47-48], whereas the estimates of $\tau_{3}$ and $\tau_{4}$ were computed using (7) and (9), respectively. These sample estimates were then used to solve for the values of shape parameters ( $c$ and $k$ ) using (a) equations (16)-(17) from [2, page 2211] and (b) (21). The solved values of $c$ and $k$ were subsequently used in (5) to superimpose the parametric plots of the Burr Type XII pdfs shown in Figure 3.

Inspection of the two panels in Figure 3 illustrates that the $L$-moment-based Type XII pdf provides a better fit to 
the sample data. The Chi-square goodness of fit statistics along with their corresponding $p$ values given in Table 3 provide evidence that the conventional moment-based Type XII distribution does not provide a good fit to the actual data, whereas the $L$-moment-based Type XII distribution fits very well. The degrees of freedom for the Chi-square goodness of fit tests were computed as $d f=5=10$ (class intervals) -4 (estimates of the parameters) -1 (sample size).

\section{L-Correlations for the Burr Type III and Type XII Distributions}

Let $Y_{j}$ and $Y_{k}$ be two random variables with cdfs $F\left(Y_{j}\right)$ and $F\left(Y_{k}\right)$, respectively. The second $L$-moments of $Y_{j}$ and $Y_{k}$ can be defined as [31]

$$
\begin{aligned}
& \lambda_{2}\left(Y_{j}\right)=2 \operatorname{Cov}\left(Y_{j}, F\left(Y_{j}\right)\right), \\
& \lambda_{2}\left(Y_{k}\right)=2 \operatorname{Cov}\left(Y_{k}, F\left(Y_{k}\right)\right) .
\end{aligned}
$$

The second $L$-comoments of $Y_{j}$ toward $Y_{k}$ and $Y_{k}$ toward $Y_{j}$ are given as

$$
\begin{aligned}
& \lambda_{2}\left(Y_{j}, Y_{k}\right)=2 \operatorname{Cov}\left(Y_{j}, F\left(Y_{k}\right)\right), \\
& \lambda_{2}\left(Y_{k}, Y_{j}\right)=2 \operatorname{Cov}\left(Y_{k}, F\left(Y_{j}\right)\right) .
\end{aligned}
$$

The $L$-correlations of $Y_{j}$ toward $Y_{k}$ and $Y_{k}$ toward $Y_{j}$ are subsequently defined as

$$
\begin{gathered}
\eta_{j k}=\frac{\lambda_{2}\left(Y_{j}, Y_{k}\right)}{\lambda_{2}\left(Y_{j}\right)}, \\
\eta_{k j}=\frac{\lambda_{2}\left(Y_{k}, Y_{j}\right)}{\lambda_{2}\left(Y_{k}\right)} .
\end{gathered}
$$

The $L$-correlation given in (25) (or, (26)) is bounded in the interval $-1 \leq \eta_{j k} \leq 1$. A value of $\eta_{j k}=1\left(\right.$ or $\left.\eta_{j k}=-1\right)$ implies that $Y_{j}$ and $Y_{k}$ have a strictly and monotonically increasing (or decreasing) relationship. See Serfling and Xiao [31] for further details on the topics related to the $L$ correlation.

The extension of the Burr Type III and Type XII distributions to multivariate level can be obtained by specifying $T$ quantile functions as given in (2) and (3) with a specified $L$ correlation structure. Specifically, let $Z_{1}, \ldots, Z_{T}$ denote standard normal variables with cdfs and the joint pdf associated with $Z_{j}$ and $Z_{k}$ given by the following expressions:

$$
\begin{aligned}
\Phi\left(Z_{j}\right)=\int_{-\infty}^{z_{j}}(2 \pi)^{-1 / 2} \exp \left\{-\frac{v_{j}^{2}}{2}\right\} d v_{j}, \\
\Phi\left(Z_{k}\right)=\int_{-\infty}^{z_{k}}(2 \pi)^{-1 / 2} \exp \left\{-\frac{v_{k}^{2}}{2}\right\} d v_{k}, \\
f_{j k}=\left(2 \pi\left(1-r_{j k}^{2}\right)^{1 / 2}\right)^{-1} \exp \left\{-\left(2\left(1-r_{j k}^{2}\right)\right)^{-1}\right. \\
\left.\times\left(z_{j}^{2}+z_{k}^{2}-2 r_{j k} z_{j} z_{k}\right)\right\},
\end{aligned}
$$

where $r_{j k}$ in (29) is the intermediate correlation (IC) between $Z_{j}$ and $Z_{k}$. Using the cdfs in (27) and (28) as zero-one uniform deviates, that is, $\Phi\left(Z_{j}\right), \Phi\left(Z_{k}\right) \sim U(0,1)$, the quantile function defined in either (2) or (3) can be ex-pressed as a function of $\Phi\left(Z_{j}\right)$, or $\Phi\left(Z_{k}\right)$ (e.g., $q_{j}\left(\Phi\left(Z_{j}\right)\right)$ or $\left.q_{k}\left(\Phi\left(Z_{k}\right)\right)\right)$. Thus, the $L$-correlation of $Y_{j}=q_{j}\left(\Phi\left(Z_{j}\right)\right)$ toward $Y_{k}=$ $q_{k}\left(\Phi\left(Z_{k}\right)\right)$ can be determined using $(25)$ with the denominator standardized to $\lambda_{2}\left(Y_{j}\right)=1 / \sqrt{\pi}$ for the standard normal distribution as

$$
\eta_{j k}=2 \sqrt{\pi} \iint_{-\infty}^{\infty} x_{j}\left(q_{j}\left(\Phi\left(Z_{j}\right)\right)\right) \Phi\left(z_{k}\right) f_{j k} d z_{j} d z_{k} .
$$

The variable $x_{j}\left(q_{j}\left(\Phi\left(Z_{j}\right)\right)\right)$ in (30) is the standardized quantile function of (2) or (3) such that it has an $L$-mean (or arithmetic mean) of zero and $L$-scale equal to that of the standard normal distribution. That is, the quantile function $Y_{j}=q_{j}\left(\Phi\left(Z_{j}\right)\right)$ is standardized by a linear transformation as

$$
x_{j}\left(q_{j}\left(\Phi\left(Z_{j}\right)\right)\right)=\delta\left(q_{j}\left(\Phi\left(Z_{j}\right)\right)-\lambda_{1}\right),
$$

where $\lambda_{1}$ is the mean from (13) or (19) and $\delta$ is a constant that scales $\lambda_{2}$ in (14) or (20) and in the denominator of (25) to $1 / \sqrt{\pi}$. In particular, $\delta$ for the Type III and Type XII distributions can be expressed as

$$
\begin{aligned}
& \delta_{\text {(Type III) }} \\
& =\frac{-\Gamma[k] \Gamma[2 k]}{\sqrt{\pi}\{\Gamma[1+1 / c](\Gamma[2 k] \Gamma[k-1 / c]-\Gamma[k] \Gamma[2 k-1 / c])\}}, \\
& \delta_{(\text {Type XII) }}(32) \\
& =\frac{\Gamma[k] \Gamma[2 k]}{\sqrt{\pi}\{\Gamma[1+1 / c](\Gamma[2 k] \Gamma[k-1 / c]-\Gamma[k] \Gamma[2 k-1 / c])\}} .
\end{aligned}
$$

The next step is to use (30) to solve for the values of the $T(T-$ 1)/2 ICs $\left(r_{j k}\right)$ such that the $T$ specified Type III and Type XII distributions have their specified $L$-correlation structure.

Analogously, the $L$-correlation of $Y_{k}=q_{k}\left(\Phi\left(Z_{k}\right)\right)$ toward $Y_{j}=q_{j}\left(\Phi\left(Z_{j}\right)\right)$ is given as

$$
\eta_{k j}=2 \sqrt{\pi} \iint_{-\infty}^{\infty} x_{k}\left(q_{k}\left(\Phi\left(Z_{k}\right)\right)\right) \Phi\left(z_{j}\right) f_{j k} d z_{k} d z_{j} .
$$

Note that in general, the $L$-correlation of $Y_{j}=q_{j}\left(\Phi\left(Z_{j}\right)\right)$ toward $Y_{k}=q_{k}\left(\Phi\left(Z_{k}\right)\right)$ in (30) is not equal to the $L$ correlation of $Y_{k}=q_{k}\left(\Phi\left(Z_{k}\right)\right)$ toward $Y_{j}=q_{j}\left(\Phi\left(Z_{j}\right)\right)$ in (34). These $L$-correlations are equal only when the values of shape parameters $c$ and $k$ associated with $q_{j}\left(\Phi\left(Z_{j}\right)\right)$ and $q_{k}\left(\Phi\left(Z_{k}\right)\right)$ are equal (i.e., when the two distributions are the same). Provided in Algorithm 1 is a source code written in Mathematica [36, 37], which shows an example for computing $\mathrm{ICs}\left(r_{j k}\right)$ for the $L$-correlation procedure. The steps for simulating correlated Burr Type III and Type XII distributions with specified values of $L$-skew $\left(\tau_{3}\right), L$-kurtosis $\left(\tau_{4}\right)$, and with specified $L$-correlation structure are given in Section 5 . 


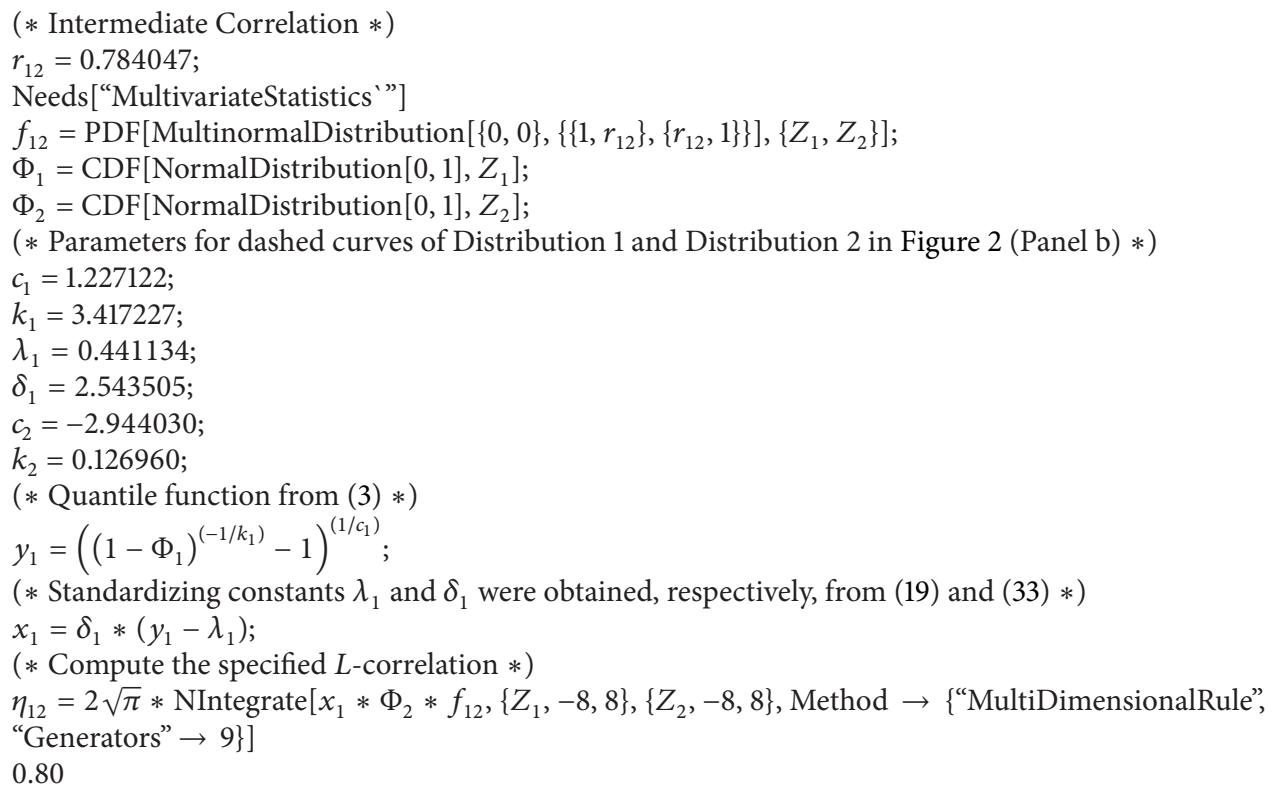

Algorithm 1: Mathematica source code for computing intermediate correlations for specified $L$-correlations. The example is for distribution $j=1$ toward distribution $k=2\left(\eta_{12}\right)$. See dashed curves of Distribution 1 and Distribution 2 in Figure 2 (Panel b), specified correlation in Table 8, and intermediate correlation in Table 10.

\section{The Procedure for Monte Carlo Simulation with an Example}

The procedure for simulating Burr Type III and Type XII distributions with specified $L$-moments and $L$-correlations can be summarized in the following six steps.

(1) Specify the $L$-moments for $T$ transformations of the form in (2) and (3), that is, $q_{1}\left(\Phi\left(z_{1}\right)\right), \ldots, q_{T}\left(\Phi\left(z_{T}\right)\right)$, and obtain the solutions for the shape parameters $c$ and $k$ by simultaneously solving the systems of equations (15) and (21) for the specified values of $L$-skew $\left(\tau_{3}\right)$ and $L$-kurtosis $\left(\tau_{4}\right)$ for each distribution. Specify a $T \times T$ matrix of $L$-correlations $\left(\eta_{j k}\right)$ for $q_{j}\left(\Phi\left(z_{j}\right)\right)$ toward $q_{k}\left(\Phi\left(Z_{k}\right)\right)$, where $j<k \in\{1,2, \ldots, T\}$.

(2) Compute the values of intermediate (Pearson) correlations (ICs), $r_{j k}$, by substituting the value of specified $L$-correlation $\left(\eta_{j k}\right)$ and the solved values of $c$ and $k$ from Step (1) into the left- and the right-hand sides of (30), respectively, and then numerically integrating (30) to solve for $r_{j k}$. See Algorithm 1 for an example. Repeat this step separately for all $T(T-1) / 2$ pairwise combinations of ICs.

(3) Assemble the ICs computed in Step (2) into a $T \times$ $T$ matrix and then decompose this matrix using Cholesky factorization. Note that this step requires the IC matrix to be positive definite.
(4) Use elements of the matrix resulting from Cholesky factorization of Step (3) to generate $T$ standard normal variables $\left(Z_{1}, \ldots, Z_{T}\right)$ correlated at the IC levels as follows:

$Z_{1}=a_{11} V_{1}$

$Z_{2}=a_{12} V_{1}+a_{22} V_{2}$

$Z_{j}=a_{1 j} V_{1}+a_{2 j} V_{2}+\cdots+a_{i j} V_{i}+\cdots+a_{j j} V_{j}$

$Z_{T}=a_{1 T} V_{1}+a_{2 T} V_{2}+\cdots+a_{i T} V_{i}+\cdots+a_{j T} V_{j}+\cdots+a_{T T} V_{T}$,

where $V_{1}, \ldots, V_{T}$ are independent standard normal random variables, and where $a_{i j}$ is the element in the $i$ th row and $j$ th column of the matrix resulting from Cholesky factorization of Step (3).

(5) Substitute $Z_{1}, \ldots, Z_{T}$ from Step (4) into the following Taylor series-based expansion for computing the cdf, $\Phi\left(Z_{j}\right)$, of standard normal distribution [38]

$\Phi\left(Z_{j}\right)$

$$
=\left(\frac{1}{2}\right)+\phi\left(Z_{j}\right)\left\{Z_{j}+\frac{Z_{j}^{3}}{3}+\frac{Z_{j}^{5}}{(3 \cdot 5)}+\frac{Z_{j}^{7}}{(3 \cdot 5 \cdot 7)}+\cdots\right\},
$$


TABLE 8: Specified correlations for the distributions in Figure 2.

\begin{tabular}{ccccc}
\hline & 1 & 2 & 3 & 4 \\
\hline 1 & 1.0 & & & \\
2 & 0.80 & 1.0 & & \\
3 & 0.75 & 0.70 & 1.0 & \\
4 & 0.60 & 0.50 & 0.40 & 1.0 \\
\hline
\end{tabular}

TABLE 9: Intermediate correlations for the conventional product moment-based (Pearson) correlation procedure.

\begin{tabular}{ccccc}
\hline & 1 & 2 & 3 & 4 \\
\hline 1 & 1.0 & & & \\
2 & 0.868005 & 1.0 & & \\
3 & 0.823239 & 0.804802 & 1.0 & \\
4 & 0.718908 & 0.646685 & 0.415208 & 1.0 \\
\hline
\end{tabular}

where $\phi\left(Z_{j}\right)$ is the pdf of standard normal distribution and the absolute error associated with (36) is less than $8 \times 10^{-16}$.

(6) Substitute the uniform $(0,1)$ variables, $\Phi\left(Z_{j}\right)$, generated in Step (5) into the $T$ equations of the form $q_{j}\left(\Phi\left(z_{j}\right)\right)$ in (2) and (3) to generate the Burr Type III and Type XII distributions with specified values of $L$-skew $\left(\tau_{3}\right), L$-kurtosis $\left(\tau_{4}\right)$, and with specified $L$ correlation structure.

For the purpose of evaluating the proposed methodology and demonstrating the steps above, an example is subsequently provided to compare the $L$-correlation-based procedure with the conventional product moment-based (Pearson) correlation procedure. Specifically, the distributions in Figure 2 (dashed curves) are used as a basis for a comparison using the specified correlation matrix in Table 8 where both strong and moderate correlations are considered in a single matrix. Let the four distributions in Figure 2 be $Y_{1}=q_{1}\left(\Phi\left(Z_{1}\right)\right), Y_{2}=q_{2}\left(\Phi\left(Z_{2}\right)\right), Y_{3}=q_{3}\left(\Phi\left(Z_{3}\right)\right)$, and $Y_{4}=q_{4}\left(\Phi\left(Z_{4}\right)\right)$, where $Y_{2}$ and $Y_{4}$ are the quantile functions from (2), and $Y_{1}$ and $Y_{3}$ are the quantile functions from (3). The specified values of conventional and $L$-moments associated with these four distributions are given in Tables 4 and 5, respectively. Presented in Tables 9 and 10 are the intermediate correlations (ICs) obtained for the conventional product moment-based (Pearson) correlation and $L$-moment-based $L$-correlation procedures, respectively, for the distributions in Figure 2. Provided in Algorithm 2 is a source code written in Mathematica [36, 37], which shows an example for computing ICs $\left(r_{j k}\right)$ for the conventional product moment-based (Pearson) correlation procedure. See also Headrick et al. [2, pages 2217-2221] for a detailed methodology for simulating correlated Burr distributions through the method of Pearson correlation.

Provided in Tables 11 and 12 are the results of Cholesky factorization on the IC matrices in Tables 9 and 10, respectively. The elements of matrices in Tables 11 and 12 are used to generate $Z_{1}, \ldots, Z_{4}$ correlated at the IC levels by making use of the formulae (35) in Step 4 with $T=4$. The values of
TABLE 10: Intermediate correlations for the $L$-moment-based $L$ correlation procedure.

\begin{tabular}{ccccc}
\hline & 1 & 2 & 3 & 4 \\
\hline 1 & 1.0 & & & \\
2 & 0.784047 & 1.0 & & \\
3 & 0.731921 & 0.679639 & 1.0 & \\
4 & 0.579134 & 0.478463 & 0.395474 & 1.0 \\
\hline
\end{tabular}

TABLE 11: Cholesky decomposition on the intermediate correlations from Table 9.

\begin{tabular}{lccc}
\hline$a_{11}=1.0$ & $a_{12}=0.868005$ & $a_{13}=0.823239$ & $a_{14}=0.718908$ \\
$a_{21}=0.0$ & $a_{22}=0.496556$ & $a_{23}=0.181704$ & $a_{24}=0.045655$ \\
$a_{31}=0.0$ & $a_{32}=0.0$ & $a_{33}=0.537830$ & $a_{34}=-0.343828$ \\
$a_{41}=0.0$ & $a_{42}=0.0$ & $a_{43}=0.0$ & $a_{44}=0.602387$ \\
\hline
\end{tabular}

TABLE 12: Cholesky decomposition on the intermediate correlations from Table 10.

\begin{tabular}{cccc}
\hline$a_{11}=1.0$ & $a_{12}=0.784047$ & $a_{13}=0.731921$ & $a_{14}=0.579134$ \\
$a_{21}=0.0$ & $a_{22}=0.620701$ & $a_{23}=0.170417$ & $a_{24}=0.039301$ \\
$a_{31}=0.0$ & $a_{32}=0.0$ & $a_{33}=0.659734$ & $a_{34}=-0.053210$ \\
$a_{41}=0.0$ & $a_{42}=0.0$ & $a_{43}=0.0$ & $a_{44}=0.812544$ \\
\hline
\end{tabular}

$Z_{1}, \ldots, Z_{4}$ are then used in (36) to obtain the Taylor seriesbased approximations of the cdfs $\Phi\left(Z_{1}\right), \Phi\left(Z_{2}\right), \Phi\left(Z_{3}\right)$, and $\Phi\left(Z_{4}\right)$, which are treated as uniform $(0,1)$ variables. These uniform variables are used in (2) and (3) to obtain the quantile functions $q_{1}\left(\Phi\left(Z_{1}\right)\right), q_{2}\left(\Phi\left(Z_{2}\right)\right), q_{3}\left(\Phi\left(Z_{3}\right)\right)$, and $q_{4}\left(\Phi\left(Z_{4}\right)\right)$ to generate the four distributions in Figure 2 that are correlated at the specified correlation level of Table 8 .

For the Monte Carlo simulation, Fortran [33] algorithm was written for both procedures to generate 25,000 independent sample estimates for the specified parameters of (a) conventional product moment-based (Pearson) correlation $\left(\rho_{j k}\right)$ and (b) $L$-moment-based $L$-correlation $\left(\eta_{j k}\right)$ based on samples of sizes $n=25$ and $n=1000$. The estimate for $\rho_{j k}$ was based on the usual formula for the Pearson correlation statistic. The estimate of $\eta_{j k}$ was computed by substituting (23) and (24) into (25), where the empirical forms of the cdfs were used in (23) and (24). The sample estimates $\rho_{j k}$ and $\eta_{j k}$ were both transformed using Fisher's $z^{\prime}$ transformations. Bias-corrected accelerated bootstrapped average estimates (Estimate), 95\% bootstrap confidence intervals (95\% Bootstrap C.I.), and standard errors (St. error) were obtained for the estimates associated with the parameters $\left(z_{\left(\rho_{j k}\right)}^{\prime}\right.$ and $\left.z_{\left(\eta_{j k}\right)}^{\prime}\right)$ using 10,000 resamples via the commercial software package Spotfire S+ [34]. The bootstrap results associated with the estimates of $z_{\left(\rho_{j k}\right)}^{\prime}$ and $z_{\left(\eta_{j k}\right)}^{\prime}$ were transformed back to their original metrics. Further, if a parameter was outside its associated $95 \%$ bootstrap C.I., then the percentage of relative bias (RB\%) was computed for the estimate as in (22). The results of this simulation are presented in Tables 13 and 14 and are discussed in Section 6. 


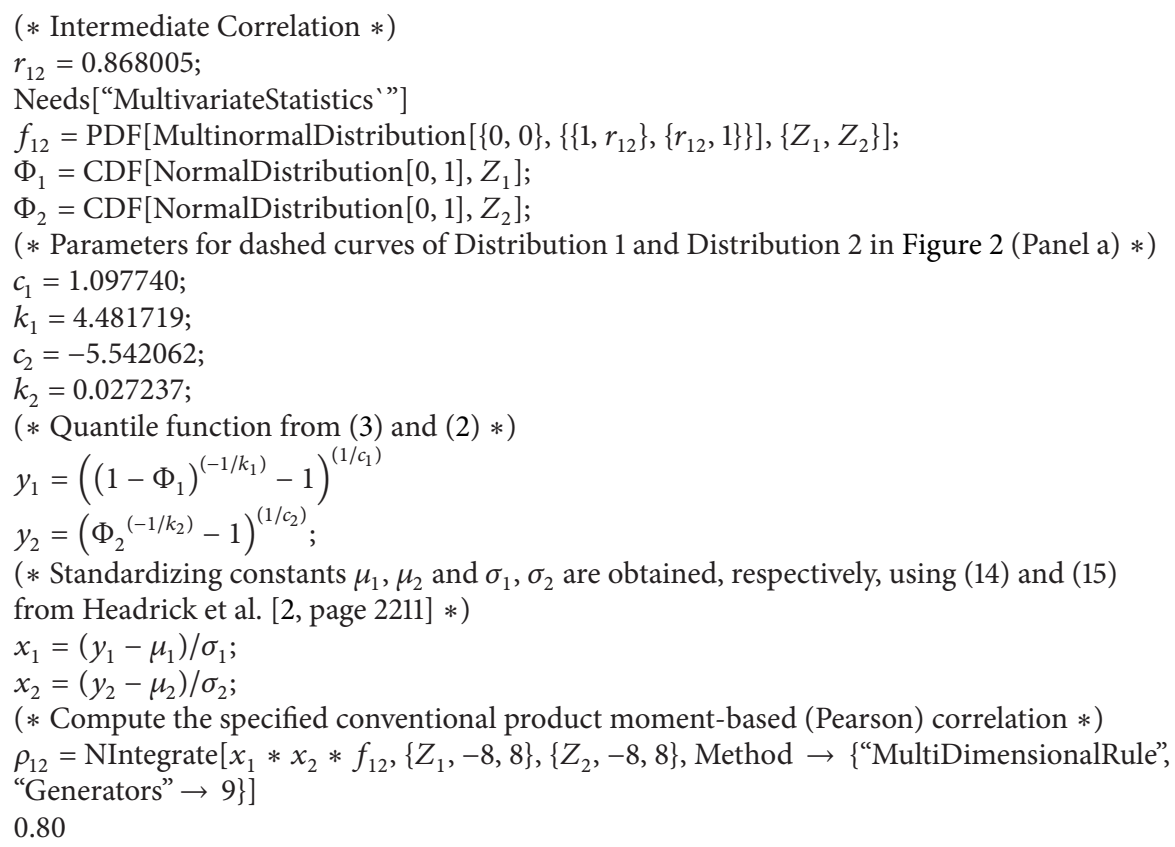

Algorithm 2: Mathematica source code for computing intermediate correlations for specified conventional product moment-based (Pearson) correlations. The example is for distribution $j=1$ and distribution $k=2\left(\rho_{12}\right)$. See dashed curves of Distribution 1 and Distribution 2 in Figure 2 (Panel a), specified correlation in Table 8 and intermediate correlation in Table 9.

TABLE 13: Correlation results for the conventional product moment-based (Pearson) correlations.

\begin{tabular}{|c|c|c|c|c|}
\hline Parameter & Estimate & 95\% Bootstrap C.I. & St. error & $\mathrm{RB} \%$ \\
\hline \multicolumn{5}{|c|}{$n=25$} \\
\hline$\rho_{12}=0.80$ & 0.8252 & $(0.8239,0.8266)$ & 0.00220 & 3.15 \\
\hline$\rho_{13}=0.75$ & 0.7910 & $(0.7899,0.7922)$ & 0.00155 & 5.47 \\
\hline$\rho_{14}=0.60$ & 0.6467 & $(0.6452,0.6481)$ & 0.00124 & 7.78 \\
\hline$\rho_{23}=0.70$ & 0.7125 & $(0.7110,0.7141)$ & 0.00161 & 1.79 \\
\hline$\rho_{24}=0.50$ & 0.5167 & $(0.5148,0.5185)$ & 0.00132 & 3.34 \\
\hline$\rho_{34}=0.40$ & 0.4117 & $(0.4094,0.4137)$ & 0.00131 & 2.93 \\
\hline \multicolumn{5}{|c|}{$n=1000$} \\
\hline$\rho_{12}=0.80$ & 0.8026 & $(0.8025,0.8031)$ & 0.00038 & 0.33 \\
\hline$\rho_{13}=0.75$ & 0.7547 & $(0.7545,0.7551)$ & 0.00034 & 0.63 \\
\hline$\rho_{14}=0.60$ & 0.6044 & $(0.6040,0.6047)$ & 0.00026 & 0.73 \\
\hline$\rho_{23}=0.70$ & 0.7003 & $(0.7001,0.7006)$ & 0.00026 & 0.04 \\
\hline$\rho_{24}=0.50$ & 0.5007 & $(0.5004,0.5010)$ & 0.00021 & 0.14 \\
\hline$\underline{\rho_{34}}=0.40$ & 0.4003 & $(0.4000,0.4006)$ & 0.00020 & 一 \\
\hline
\end{tabular}

\section{Discussion and Conclusion}

One of the advantages that $L$-moments have over conventional moments can be expressed in the context of estimation. The $L$-moment-based estimators of $L$-skew and $L$-kurtosis can be far less biased than the conventional moment-based estimators of skew and kurtosis when samples are drawn from the distributions with more severe departures from normality [18, 25-29, 31]. Inspection of the simulation results in Tables 6 and 7 clearly indicates that this is the case for the Burr Type III and Type XII distributions. That is, the superiority that estimates of $L$-moment ratios $\left(\tau_{3}\right.$ and $\left.\tau_{4}\right)$ have over their corresponding conventional moment-based estimates of skew and kurtosis $\left(\gamma_{3}\right.$ and $\left.\gamma_{4}\right)$ is obvious. For example, for samples of size $n=25$, the estimates of $\gamma_{3}$ and $\gamma_{4}$ for Distribution 1 were, on average, $41.46 \%$ and $5.62 \%$ of their associated parameters, whereas the estimates of $\tau_{3}$ and $\tau_{4}$ were $93.88 \%$ and $92.03 \%$ of their associated parameters. This advantage of $L$-moment-based estimates can also be expressed by comparing their relative standard errors (RSEs), where RSE $=\{($ St. error $/$ Estimate $) \times 100\}$. Comparing Tables 6 and 7, it is evident that the estimates of $\tau_{3}$ and $\tau_{4}$ are more 
TABLE 14: Correlation results for the $L$-moment-based $L$-correlation procedure.

\begin{tabular}{|c|c|c|c|c|}
\hline Parameter & Estimate & 95\% Bootstrap C.I. & St. error & $\mathrm{RB} \%$ \\
\hline \multicolumn{5}{|c|}{$n=25$} \\
\hline$\eta_{12}=0.80$ & 0.8062 & $(0.8048,0.8072)$ & 0.00172 & 0.78 \\
\hline$\eta_{13}=0.75$ & 0.7571 & $(0.7556,0.7585)$ & 0.00172 & 0.95 \\
\hline$\eta_{14}=0.60$ & 0.6047 & $(0.6026,0.6068)$ & 0.00167 & 0.78 \\
\hline$\eta_{23}=0.70$ & 0.7086 & $(0.7070,0.7105)$ & 0.00180 & 1.23 \\
\hline$\eta_{24}=0.50$ & 0.5057 & $(0.5031,0.5082)$ & 0.00176 & 1.14 \\
\hline$\eta_{34}=0.40$ & 0.4054 & $(0.4030,0.4078)$ & 0.00145 & 1.35 \\
\hline \multicolumn{5}{|c|}{$n=1000$} \\
\hline$\eta_{12}=0.80$ & 0.8001 & $(0.8000,0.8003)$ & 0.00026 & - \\
\hline$\eta_{13}=0.75$ & 0.7502 & $(0.7500,0.7504)$ & 0.00026 & - \\
\hline$\eta_{14}=0.60$ & 0.6001 & $(0.5997,0.6004)$ & 0.00026 & - \\
\hline$\eta_{23}=0.70$ & 0.7002 & $(0.6999,0.7004)$ & 0.00028 & - \\
\hline$\eta_{24}=0.50$ & 0.5002 & $(0.4998,0.5006)$ & 0.00028 & - \\
\hline$\eta_{34}=0.40$ & 0.4003 & $(0.3999,0.4006)$ & 0.00022 & - \\
\hline
\end{tabular}

efficient as their RSEs are considerably smaller than the RSEs associated with the conventional moment-based estimates of $\gamma_{3}$ and $\gamma_{4}$. For example, in terms of Distribution 1 in Figure 2, inspection of Tables 6 and 7 (for $n=1000$ ) indicates that RSE measures of RSE $\left(t_{3}\right)=0.036 \%$ and $\operatorname{RSE}\left(t_{4}\right)=0.064 \%$ are considerably smaller than the RSE measures of RSE $\left(g_{3}\right)=$ $0.270 \%$ and RSE $\left(g_{4}\right)=0.851 \%$. This demonstrates that the estimates of $L$-skew and $L$-kurtosis have more precision because they have less variance around their bootstrapped estimates.

Another advantage of $L$-moments can be highlighted in the context of distribution fitting. Comparison of the four distributions in Figures 2(a) and 2(b) clearly indicates that $L$-moment-based Burr Type III and Type XII distributions provide a better fit to the theoretical distributions compared with their conventional moment-based counterparts. This advantage is most pronounced in the context of the first two distributions: Distribution 1 and Distribution 2, where $L$-moment-based Burr Type XII and Type III in Figure 2(b) provide a better fit to the $F$ distribution $(3,10)$ and Chi-square $(d f=1)$ distributions than their conventional moment-based counterparts in Figure 2(a). In the context of fitting realworld data, the $L$-moment-based Burr Type XII in Figure 3(b) provides a better fit to the ankle circumference data than the conventional moment-based Burr Type XII in Figure 3(a).

Presented in Tables 13 and 14 are the simulation results of conventional product moment-based (Pearson) correlations and $L$-moment-based $L$-correlations, respectively. Overall inspection of these tables indicates that the $L$-correlation is superior to Pearson correlation in terms of relative bias. For example, for $n=25$, the percentage of relative bias for the two distributions, Distribution 1 and Distribution 4, in Figure 2 was $7.78 \%$ for the Pearson correlation compared with only $0.78 \%$ for the $L$-correlation. It is also noted that the variability associated with bootstrapped estimates of $L$-correlation appears to be more stable than that of the bootstrapped estimates of Pearson correlation both within and across different conditions.
In summary, the new $L$-moment-based procedure is an attractive alternative to the more traditional conventional moment-based procedure in the context of Burr Type III and Type XII distributions. In particular, the $L$-moment-based procedure has distinct advantages when distributions with large departures from normality are used. Finally, we note that Mathematica $[36,37]$ source codes are available from the authors for implementing both the conventional momentand $L$-moment-based procedures.

\section{References}

[1] I. W. Burr, "Cumulative frequency functions," Annals of Mathematical Statistics, vol. 13, pp. 215-232, 1942.

[2] T. C. Headrick, M. D. Pant, and Y. Sheng, "On simulating univariate and multivariate Burr Type III and Type XII distributions," Applied Mathematical Sciences, vol. 4, no. 45-48, pp. 2207-2240, 2010.

[3] I. W. Burr, "Parameters for a general system of distributions to match a grid of $\alpha_{3}$ and $\alpha_{4}$," Communications in Statistics, vol. 2, pp. 1-21, 1973.

[4] R. N. Rodriguez, "A guide to the Burr Type XII distributions," Biometrika, vol. 64, no. 1, pp. 129-134, 1977.

[5] P. R. Tadikamalla, "A look at the Burr and related distributions," International Statistical Review, vol. 48, no. 3, pp. 337-344, 1980.

[6] J. H. Gove, M. J. Ducey, W. B. Leak, and L. Zhang, "Rotated sigmoid structures in managed uneven-aged northern hardwood stands: a look at the Burr Type III distribution," Forestry, vol. 81, no. 2, pp. 161-176, 2008.

[7] S. R. Lindsay, G. R. Wood, and R. C. Woollons, "Modelling the diameter distribution of forest stands using the Burr distribution," Journal of Applied Statistics, vol. 23, no. 6, pp. 609619, 1996.

[8] S. Nadarajah and S. Kotz, " $q$ exponential is a Burr distribution," Physics Letters A, vol. 359, no. 6, pp. 577-579, 2006.

[9] S. Nadarajah and S. Kotz, "On the alternative to the Weibull function," Engineering Fracture Mechanics, vol. 74, no. 3, pp. 451-456, 2007. 
[10] D. R. Wingo, "Maximum likelihood methods for fitting the Burr type XII distribution to life test data," Biometrical Journal, vol. 25, no. 1, pp. 77-84, 1983.

[11] D. R. Wingo, "Maximum likelihood methods for fitting the Burr type XII distribution to multiply (progressively) censored life test data," Metrika, vol. 40, no. 3-4, pp. 203-210, 1993.

[12] A. S. Chernobai, F. J. Fabozzi, and S. T. Rachev, Operational Risk: A Guide to Basel II Capital Requirements, Models, and Analysis, John Wiley \& Sons, New York, NY, USA, 2007.

[13] B. J. Sherrick, P. Garcia, and V. Tirupattur, "Recovering probabilistic information from option markets: tests of distributional assumptions," Journal of Futures Markets, vol. 16, no. 5, pp. 545$560,1996$.

[14] P. W. Mielke, "Another family of distributions for describing and analyzing precipitation data," Journal of Applied Meteorology, vol. 12, pp. 275-280, 1973.

[15] H. A. Tejeda and B. K. Goodwin, "Modeling crop prices through a Burr distribution and analysis of correlation between crop prices and yields using a copula method," Paper presented at the annual meeting of the Agricultural and Applied Economics Association, Orlando, FL, USA, 2008, http://purl.umn.edu/ 6061.

[16] A. A. Abdel-Ghaly, G. R. Al-Dayian, and F. H. Al-Kashkari, “The use of burr type XII distribution on software reliability growth modelling," Microelectronics Reliability, vol. 37, no. 2, pp. 305313, 1997.

[17] N. A. Mokhlis, "Reliability of a stress-strength model with Burr Type III distributions," Communications in Statistics, vol. 34, no. 7, pp. 1643-1657, 2005.

[18] T. C. Headrick, "A characterization of power method transformations through L-moments," Journal of Probability and Statistics, Article ID 497463, 22 pages, 2011.

[19] J. R. M. Hosking, " $L$-moments: analysis and estimation of distributions using linear combinations of order statistics," Journal of the Royal Statistical Society B, vol. 52, no. 1, pp. 105$124,1990$.

[20] U. H. Olsson, T. Foss, and S. V. Troye, "Does the ADF fit function decrease when the kurtosis increases?" The British Journal of Mathematical and Statistical Psychology, vol. 56, no. 2, pp. 289303, 2003.

[21] I. Berkovits, G. R. Hancock, and J. Nevitt, "Bootstrap resampling approaches for repeated measure designs: relative robustness to sphericity and normality violations," Educational and Psychological Measurement, vol. 60, no. 6, pp. 877-892, 2000.

[22] C. K. Enders, "The impact of nonnormality on full information maximum-likelihood estimation for structural equation models with missing data," Psychological Methods, vol. 6, no. 3, pp. 352-370, 2001.

[23] M. Kendall and A. Stuart, The Advanced Theory of Statistics, Macmillan, New York, NY, USA, 4th edition, 1977.

[24] J. R. M. Hosking, "Moments or L-moments? An example comparing two measures of distributional shape," American Statistician, vol. 46, no. 3, pp. 186-189, 1992.

[25] J. R. M. Hosking and J. R. Wallis, Regional Frequency Analysis: An Approach Based on L-Moments, Cambridge University Press, Cambridge, UK, 1997.

[26] T. C. Headrick and M. D. Pant, "Simulating non-normal distributions with specified $L$-moments and $L$-correlations," Statistica Neerlandica, vol. 66, no. 4, pp. 422-441, 2012.

[27] T. C. Headrick and M. D. Pant, "A method for simulating nonnormal distributions with specified $L$-skew, $L$-kurtosis, and
L-correlation," ISRN Applied Mathematics, Article ID 980827, 23 pages, 2012.

[28] T. C. Headrick and M. D. Pant, "A logistic $L$-moment-based analog for the Tukey $g-h, g, h$, and $h$ - $h$ system of distributions," ISRN Probability and Statistics, vol. 2012, Article ID 245986, 23 pages, 2012.

[29] T. C. Headrick and M. D. Pant, "An L-moment-based analog for the Schmeiser-Deutsch class of distributions," ISRN Applied Mathematics, vol. 2012, Article ID 475781, 16 pages, 2012.

[30] J. Karvanen and A. Nuutinen, "Characterizing the generalized lambda distribution by $L$-moments," Computational Statistics \& Data Analysis, vol. 52, no. 4, pp. 1971-1983, 2008.

[31] R. Serfling and P. Xiao, "A contribution to multivariate $L$ moments: L-comoment matrices," Journal of Multivariate Analysis, vol. 98, no. 9, pp. 1765-1781, 2007.

[32] M. C. Jones, "On some expressions for variance, covariance, skewness and L-moments," Journal of Statistical Planning and Inference, vol. 126, no. 1, pp. 97-106, 2004.

[33] "Microsoft FORTRAN PowerStation Version 4. 0. Microsoft Corporation, Redmond, Wash, USA," 1994.

[34] "TIBCO Spotfire S+ 8. 2. 0 for Microsoft Windows. TIBCO Software Inc., Palo Alto, Calif, USA," 2010.

[35] T. C. Headrick, Statistical Simulation: Power Method Polynomials and Other Transformations, Chapman \& Hall/CRC, Boca Raton, Fla, USA, 2010.

[36] "Wolfram Mathematica 8. 0. 4. 0 for Microsoft Windows. Wolfram Research, Inc., Champaign, Ill, USA," 2011.

[37] S. Wolfram, The Mathematica Book, Wolfram Media, Champaign, Ill, USA, 4th edition, 1999.

[38] G. Marsaglia, "Evaluating the normal distribution function," Journal of Statistical Software, vol. 11, pp. 1-10, 2004. 


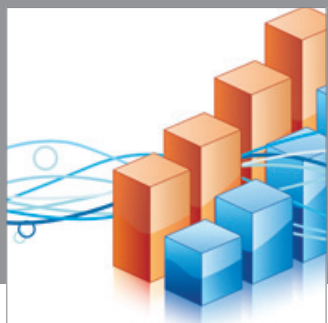

Advances in

Operations Research

mansans

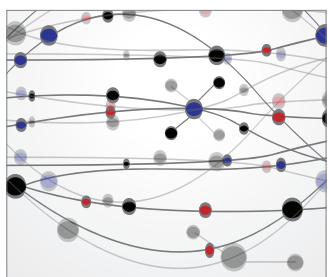

The Scientific World Journal
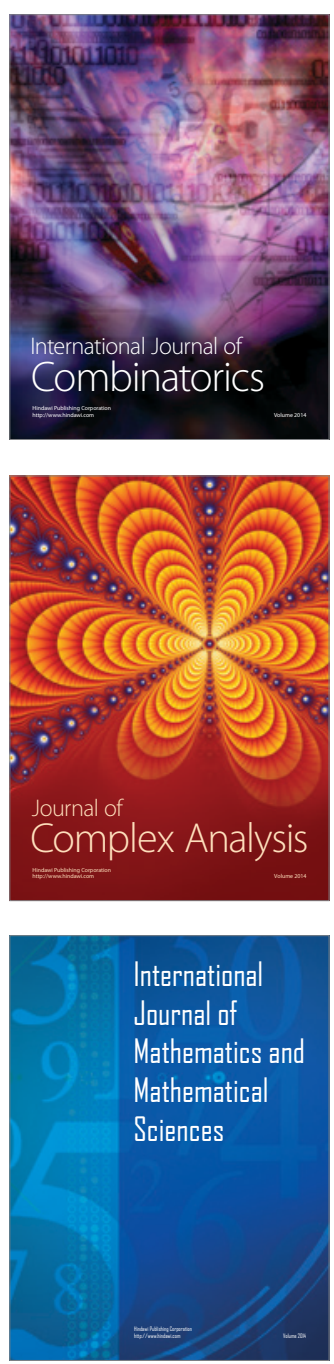
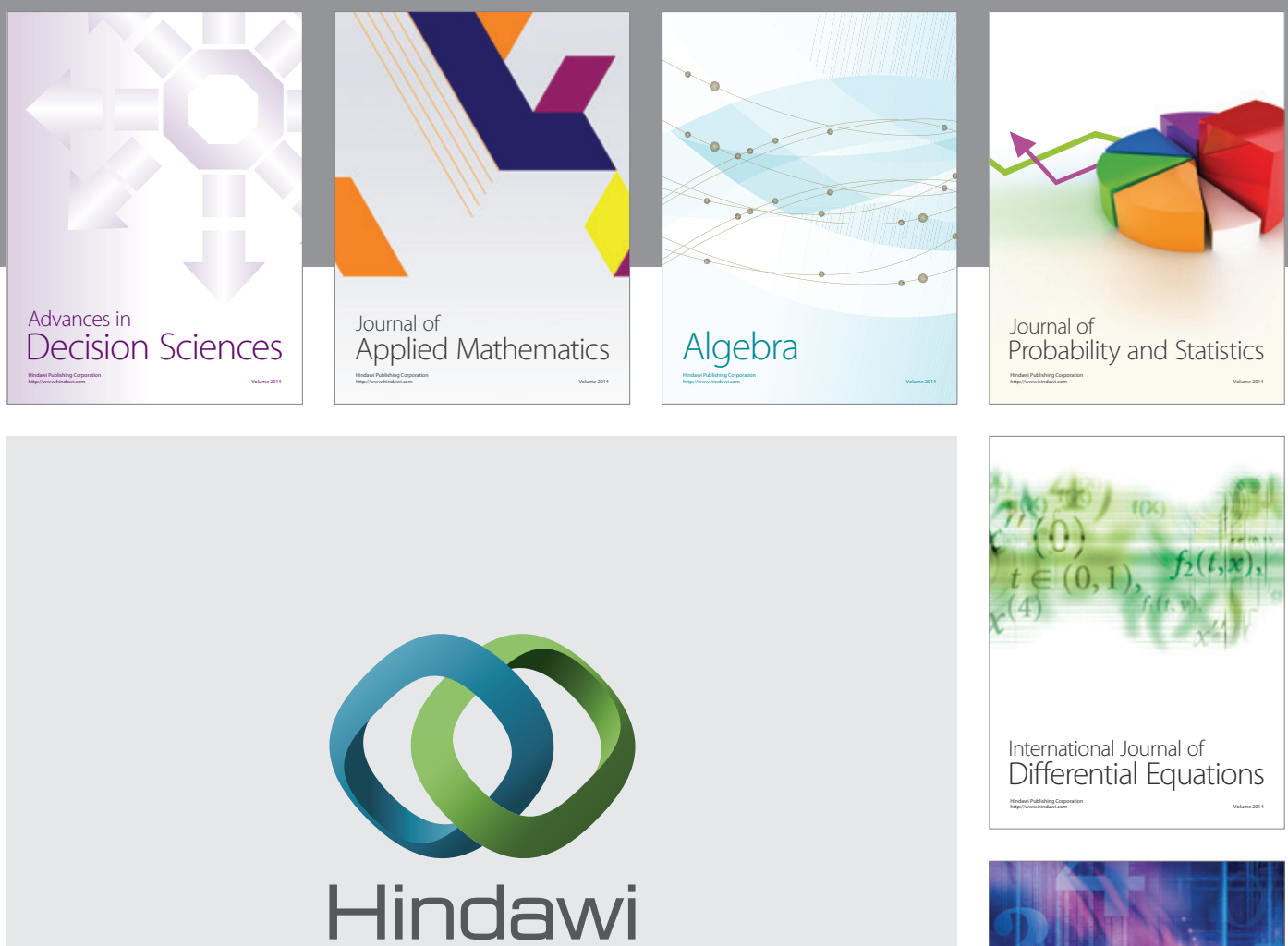

Submit your manuscripts at http://www.hindawi.com
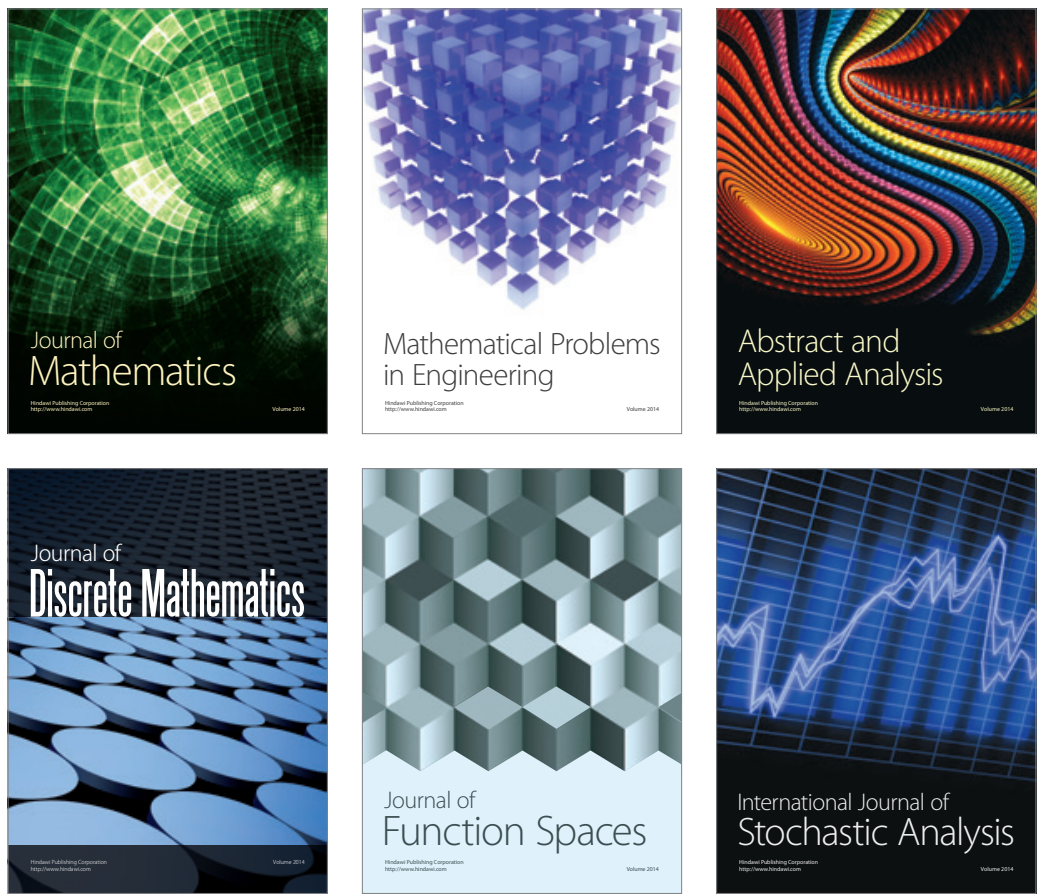

Journal of

Function Spaces

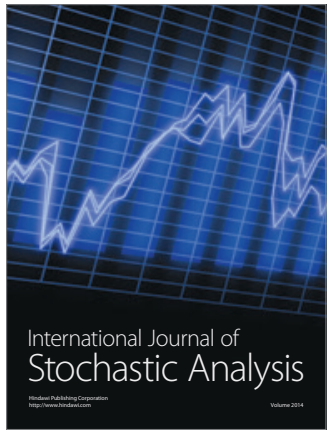

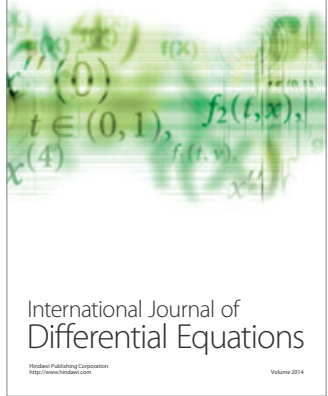
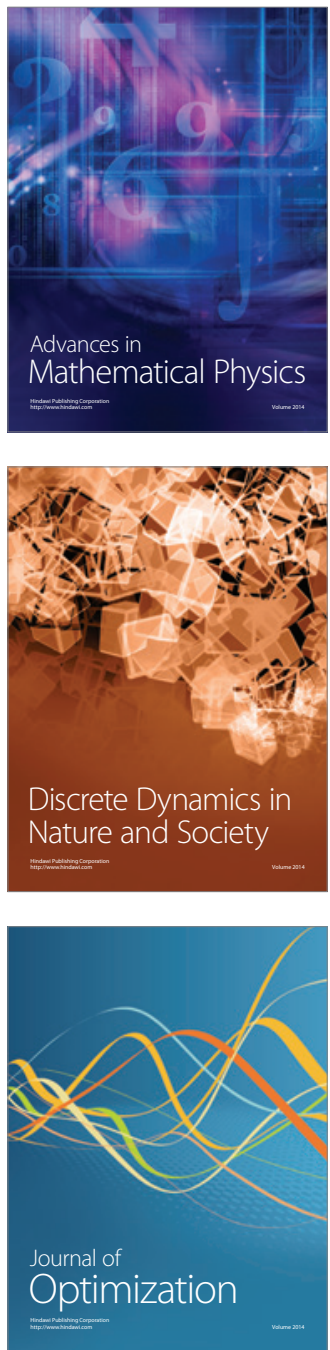